\title{
TRACE CLASS PERTURBATIONS OF ISOMETRIES AND UNITARY DILATIONS
}

\author{
RICHARD W. CAREY
}

ABSTRACT. Let $U$ and $V$ be isometries acting on a Hilbert space. If $U-V$ belongs to the trace class we show that the absolutely continuous parts of the corresponding minimal unitary dilations are unitarily equivalent.

Introduction. With $X$ and $Y$ complex Hilbert spaces let $\mathcal{B}(X, Y)$ denote the collection of bounded linear operators from $X$ to $Y$; the symbol $\mathscr{T}(X)$ will represent the trace class elements in $\mathcal{B}(X, X)$. If $A$ is a (not necessarily bounded) selfadjoint operator in $X$ with the spectral representation $A=\int_{R} \lambda d E_{\lambda}$ and $x \in X$, then $\mu_{x}(\Delta)=\|E(\Delta) x\|^{2}$ is a nonnegative, completely additive measure defined for Borel sets $\Delta$. If this measure is absolutely continuous with respect to Lebesgue measure, we say that $x$ is absolutely continuous with respect to $A$. The collection $X(A)_{\text {ac }}$ of all vectors $x$ for which the measure $\mu_{x}(\cdot)$ is absolutely continuous is a closed subspace which reduces $A$ [4], and the restriction of $A$ to this space, $A_{\text {ac }}$, is called the absolutely continuous part of $A$. A similar definition applies to a unitary operator $A$.

1. An important result on the perturbation of continuous spectra was given by Rosenblum [1] and Kato [2] and later generalized by Kuroda [3]. This theorem asserts that if $\{A, B\}$ is a pair of selfadjoint operators in a Hilbert space $X$ such that $(A-z)^{-1}-(B-z)^{-1} \in \mathfrak{T}(X)$ for some $z$ with $\operatorname{Im} z \neq 0$, then $A_{\text {ac }}$ is unitarily equivalent to $B_{\text {ac }}$. By taking Cayley transforms this may be rephrased in terms of unitary operators, i.e., if $U$ and $V$ are unitary operators for which $U-V \in \mathfrak{T}(X)$, then $U_{\text {ac }}$ and $V_{\text {ac }}$ are unitarily equivalent. The object of this note is to prove an extension of this fact for pairs of isometries. Precisely, we prove the following:

Theorem. Let $U$ and $V$ be isometries in $X$ with minimal unitary dilations

Received by the editors December 19, 1972.

AMS (MOS) subject classifications (1970). Primary 47A55, 47A20.

Key words and phrases. Isometry, unitary dilation, trace class. 
$U^{\prime}$ and $V^{\prime}$, respectively. If $U-V \in \mathcal{T}(X)$, then $U_{\mathrm{ac}}^{\prime}$ and $V_{\mathrm{ac}}^{\prime}$ are unitarily equivalent.

Proof. Since $U-V \in \mathfrak{T}(X)$, it is certainly compact; thus it suffices to consider the case of a separable space $X$. Again, since $U-V$ is compact, a property from index theory [5] says that $\operatorname{index}(U)=\operatorname{index}(V)$. But index $(I)=-$ dimension $R(I)^{\perp}(R(I) \equiv$ range $I)$, for any isometry $I$ on $X$; hence dimension $R(U)^{\perp}=$ dimension $R(V)^{\perp}$, (the symbol $\perp$ denotes orthogonal complement). Let $\alpha$ equal this common value. If $\alpha$ is infinite, then both $U_{\mathrm{ac}}^{\prime}$ and $V_{\text {ac }}^{\prime}$ have spectral multiplicity uniformly $\boldsymbol{X}$ over the entire unit circle [6, Theorem 1, p. 271] and therefore $U_{\mathrm{ac}}^{\prime}$ is unitarily equivalent to $V_{\mathrm{ac}}^{\prime}$. It remains to consider the case when $\alpha$ is a finite number.

Our strategy consists in extending $U$ and $V$ to minimal unitary dilations $U^{\prime}$ and $V^{\prime}$ on the same dilation space $X^{\prime}$ in such a manner that $U^{\prime}-V^{\prime} \epsilon$ $\mathcal{T}\left(X^{\prime}\right)$. The assertion of the Theorem will then follow by invoking the result of Rosenblum-Kato-Kuroda for the pair $\left\{U^{\prime}, V^{\prime}\right\}$.

The construction uses the Wold decomposition $[6$, The orem 1.1, p. 3]: Let

$$
\begin{aligned}
& U \cong S_{\alpha} \oplus U_{u}, \\
& V \cong S_{\alpha} \oplus V_{u}
\end{aligned}
$$

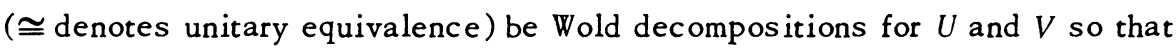
$S_{a}$ is a unilateral shift of multiplicity $\alpha$, and $\left\{U_{u}, V_{u}\right\}$ are unitary operators not necessarily acting on the same space. Let $b$ denote the $l_{2}$ space with dimension $\alpha$. Without loss of generality we may assume that our original space $X$ decomposes into an orthogonal sum $X_{a} \oplus X_{u}(U)$ such that $X_{a}$ is the Hilbert space whose elements are vector functions $\xi=\left\langle\xi_{0}, \xi_{1}, \xi_{2}, \ldots\right\rangle$ with $\xi_{n} \in h,\|\xi\|^{2}=\Sigma_{n=0}^{\infty}\left\|\xi_{n}\right\|^{2}<\infty$. The operator $S_{a}$ acts on $X_{a}$ by $S_{a} \xi=$ $\left\langle\eta_{0}, \eta_{1}, \eta_{2}, \cdots\right\rangle ; \eta_{0}=0, \eta_{n}=\xi_{n-1}, n \geq 1$, and $U_{u}$ operates in $X_{u}(U)$. Moreover, with $V_{u}$ operating in the space $X_{u}(V)$, the decomposition (2) implies the existence of an isometric map $\phi$ of $Y \equiv X_{\alpha} \oplus X_{u}(V)$ onto $X$ such that $V=$ $\phi\left(S_{a} \oplus V_{u}\right) \phi^{*}$, where $\phi^{*} \in \mathfrak{B}(X, Y)$ is the adjoint map. Let us define dilation spaces by setting

$$
\begin{aligned}
& X^{\prime}=\hat{X}_{a} \oplus^{\prime} X \equiv \hat{X}_{a} \oplus^{\prime} X_{a} \oplus X_{u}(U), \\
& Y^{\prime}=\hat{X}_{\alpha} \oplus^{\prime} Y \equiv \hat{X}_{a} \oplus^{\prime} X_{a} \oplus X_{u}(V)
\end{aligned}
$$

where $\hat{X}_{a}$ is the Hilbert space whose elements are the vectors $\xi=$ $\left\langle\cdots, \xi_{-3}, \xi_{-2}, \xi_{-1}\right\rangle$ with $\xi_{n} \in h,\|\xi\|^{2}=\Sigma_{n=1}^{\infty}\left\|\xi_{-n}\right\|^{2}<\infty$, and $\oplus^{\prime}$ denotes orthogonal direct sum. A minimal unitary dilation of $U$ is then given by the 
operator $U^{\prime} \in \mathcal{B}\left(X^{\prime}, X^{\prime}\right)$ defined as follows [6, Chapter $\left.1, \S 2\right]$ : If

$$
x^{\prime}=\left\langle\cdots, \xi_{-3}, \xi_{-2}, \xi_{-1}\right\rangle \oplus^{\prime}\left\langle\xi_{0}, \xi_{1}, \xi_{2}, \cdots\right\rangle \oplus x_{u},
$$

set

$$
U^{\prime} x^{\prime}=\left\langle\cdots, \xi_{-4}, \xi_{-3}, \xi_{-2}\right\rangle \oplus^{\prime}\left\langle\xi_{-1}, \xi_{0}, \xi_{1}, \cdots\right\rangle \oplus U_{u} x_{u}
$$

Further, we construct a minimal unitary dilation $V^{\prime}$ of $V$ by setting $V^{\prime}=$ $\phi^{\prime} M^{\prime} \phi^{\prime *}$ where $M^{\prime} \in \mathcal{B}\left(Y^{\prime}, Y^{\prime}\right) ; \phi^{\prime} \in \mathcal{B}\left(Y^{\prime}, X^{\prime}\right), \phi^{\prime *} \in \mathcal{B}\left(X^{\prime}, Y^{\prime}\right)$ are defined by

$$
\begin{aligned}
& M^{\prime} y^{\prime}=\left\langle\ldots, \xi_{-4}, \xi_{-3}, \xi_{-2}\right\rangle \oplus^{\prime}\left\langle\xi_{-1}, \xi_{0}, \xi_{1}, \ldots\right\rangle \oplus v_{u} y_{u}, \\
& \phi^{\prime} y^{\prime}=\left\langle\cdots, \xi_{-3}, \xi_{-2}, \xi_{-1}\right\rangle \oplus^{\prime} \phi\left(\left\langle\xi_{0}, \xi_{1}, \xi_{2}, \ldots\right\rangle \oplus y_{u}\right)
\end{aligned}
$$

for

$$
y^{\prime}=\left\langle\cdots, \xi_{-3}, \xi_{-2}, \xi_{-1}\right\rangle \oplus^{\prime}\left\langle\xi_{0}, \xi_{1}, \xi_{2}, \ldots\right\rangle \oplus y_{u}
$$

and $\phi^{\prime *}$ is the adjoint of $\phi^{\prime}$.

We shall now prove that $U^{\prime}-V^{\prime} \in \mathcal{T}\left(X^{\prime}\right)$. For convenience we introduce the following operators: With

$$
x^{\prime}=\xi^{(1)} \oplus^{\prime} \xi^{(2)} \oplus x \in X^{\prime} ; \quad \xi^{(1)}=\left\langle\ldots, \xi_{-3}, \xi_{-2}, \xi_{-1}\right\rangle \in \hat{X}_{a},
$$

put

$$
\begin{aligned}
P x^{\prime} & =\xi^{(2)} \oplus x \in X, \\
P_{-1} x^{\prime} & =0 \oplus^{\prime}\left\langle\xi_{-1}, 0,0, \ldots\right\rangle \oplus 0 \in X^{\prime}, \\
Q_{-1} x^{\prime} & =0 \oplus^{\prime}\left\langle\xi_{-1}, 0,0, \ldots\right\rangle \oplus 0 \in Y^{\prime}
\end{aligned}
$$

so that $P \in \mathfrak{B}\left(X^{\prime}, X\right), P_{-1} \in \mathfrak{B}\left(X^{\prime}, X^{\prime}\right)$ and $Q_{-1} \in \mathfrak{B}\left(X^{\prime}, Y^{\prime}\right)$. Note that $P$ is a projection, whereas $P_{-1}$ and $Q_{-1}$ are shifts followed by projections of rank $\alpha$; in particular, $P_{-1} \in \mathfrak{T}\left(X^{\prime}\right)$. We also introduce the injection operator of $X$ into $X^{\prime}$ by setting $I x=0 \oplus^{\prime} x \in X^{\prime}$ so $I \in \mathcal{B}\left(X, X^{\prime}\right)$.

With the se definitions we shall calculate that

$$
U^{\prime}-V^{\prime}=P_{-1}+I(U-V) P-\phi^{\prime *} Q_{-1} \text {. }
$$

Choose $x^{\prime}=\xi^{(1)} \oplus^{\prime} \xi^{(2)} \oplus x$ as above. We then have

$$
\begin{aligned}
\left(U^{\prime}-V^{\prime}\right) x^{\prime} & =U^{\prime} x^{\prime}-V^{\prime} x^{\prime}=U^{\prime} x^{\prime}-\phi^{\prime} M^{\prime} \phi^{\prime *} x^{\prime} \\
& =\eta^{(1)} \oplus^{\prime} \eta^{(2)} \oplus U_{u} x-\phi^{\prime} M^{\prime}\left[\xi^{(1)} \oplus^{\prime} \phi^{*}\left(\xi^{(2)} \oplus x\right)\right]
\end{aligned}
$$

where

$$
\eta^{(1)}=\left\langle\cdots, \xi_{-4}, \xi_{-3}, \xi_{-2}\right\rangle, \quad \eta^{(2)}=\left\langle\xi_{-1}, \xi_{0}, \xi_{1}, \cdots\right\rangle .
$$


Observe that

$$
\eta^{(1)} \oplus^{\prime} \eta^{(2)} \oplus U_{u} x=\eta^{(1)} \oplus^{\prime} \tau^{(1)} \oplus 0+0 \oplus^{\prime} U\left(\xi^{(2)} \oplus x\right)
$$

where $\tau^{(1)}=\left\langle\xi_{-1}, 0,0, \cdots\right\rangle$. Also,

$$
\begin{aligned}
\phi^{\prime} M^{\prime}\left[\xi^{(1)} \oplus^{\prime} \phi^{*}\left(\xi^{(2)} \oplus x\right)\right]= & \phi^{\prime}\left[\eta^{(1)} \oplus^{\prime} 0 \oplus 0+0 \oplus^{\prime} \tau^{(1)} \oplus 0\right] \\
& +\phi^{\prime} M^{\prime}\left[0 \oplus^{\prime} \phi^{*}\left(\xi^{(2)} \oplus x\right)\right] .
\end{aligned}
$$

But

$$
\begin{gathered}
\phi^{\prime}\left(\eta^{(1)} \oplus^{\prime} 0 \oplus 0\right)=\eta^{(1)} \oplus^{\prime} 0 \oplus 0 \in X^{\prime}, \\
\phi^{\prime}\left(0 \oplus^{\prime} \tau^{(1)} \oplus 0\right)=0 \oplus^{\prime} \phi\left(\tau^{(1)} \oplus 0\right) \in X^{\prime}, \\
\phi^{\prime} M^{\prime}\left[0 \oplus^{\prime} \phi^{*}\left(\xi^{(2)} \oplus x\right)\right]=0 \oplus^{\prime} V\left(\xi^{(2)} \oplus x\right) \in X^{\prime} .
\end{gathered}
$$

Thus,

$$
\left(U^{\prime}-V^{\prime}\right) x^{\prime}=P_{-1} x^{\prime}+I(U-V) P x^{\prime}-\phi^{\prime} Q_{-1} x^{\prime}
$$

and therefore

$$
U^{\prime}-V^{\prime}=P_{-1}+I(U-V) P-\phi^{\prime} Q_{-1}
$$

as operators on $X^{\prime}$. By what has been noted above, $P_{-1}$ and $\phi^{\prime} Q_{-1} \epsilon$ $\mathscr{T}\left(X^{\prime}\right)$; since $U-V \in \mathcal{T}(X)$ by hypothesis, it follows that $I(U-V) P \in \mathcal{T}\left(X^{\prime}\right)$. We may therefore conclude that $U^{\prime}-V^{\prime} \in \mathfrak{T}\left(X^{\prime}\right)$. The result of RosenblumKato-Kuroda mentioned previously now allows us to assert that $U_{\text {ac }}^{\prime}$ is unitarily equivalent to $V_{\mathrm{ac}}^{\prime}$. This completes the proof.

Corollary 1. Let $U$ and $V$ be isometries on $X$ with the Wold decompositions $U \cong S_{a} \oplus U_{u}$ and $V \cong S_{\alpha} \oplus V_{u}$. If $U-V \in \mathcal{T}(X)$ and $\alpha$ is a finite number, then $U_{u \mathrm{ac}}$ and $V_{u \mathrm{ac}}$ are unitarily equivalent.

Proof. From the Theorem it follows that the dilations $U^{\prime}, V^{\prime}$ have unitarily equivalent absolutely continuous parts. If $B_{a}$ is the bilateral shift of multiplicity $a$, then

$$
U_{\mathrm{ac}}^{\prime}=\left(B_{\alpha} \oplus U_{u}\right)_{\text {ac }} \cong B_{a} \oplus U_{u a c}
$$

and

$$
V_{\mathrm{ac}}^{\prime} \cong\left(B_{a} \oplus V_{u}\right)_{\mathrm{ac}} \cong B_{a} \oplus V_{u \mathrm{ac}}
$$

Since $a$ is finite we deduce that $U_{u \text { ac }} \cong V_{u \text { ac }}$.

Does the conclusion of this corollary remain valid without assuming that $\alpha$ is finite? We do not know the answer to this question. However, we can prove the following result. 
Proposition. Let $U$ and $V$ be isometries on $X$ such that $U-V$ has finite rank. Then $U_{u \text { ac }}$ and $V_{v \text { ac }}$ are unitarily equivalent.

Proof. Let $U-V$ have the Schmidt series $\sum_{n=1}^{d} \phi_{n} \otimes \psi_{n}$ where $d=$ dimension $R(U-V)$ is finite. Let $M$ denote the smallest (closed) subspace of $X$ which is invariant for $U$ and contains the vectors $\phi_{1}, \phi_{2}, \cdots, \phi_{d}$. Then $\mathbb{M}$ is a lso invariant for $V$. Hence, $\mathbb{M}^{\perp}$ is invariant for $U^{*}$ and $V^{*}$. Moreover, the restriction operators on $\mathbb{M}^{\perp}$ induced by $U^{*}$ and $V^{*}$ are equal. It then follows that the ir unitary parts, if any, are also equal. Hence, $U$ and $V$ have the same unitary parts on $\pi^{\perp}$. On the other hand, the operators $U$ and $V$ restricted to $M$ have index $\geq-d$ and so by Corollary 1 we may conclude that their unitary parts have the same absolutely continuous pieces. Now, by the Wold decomposition it is clear that a vector $x$ belongs to the unitary part of an isometry $I$ provided $\left\|I^{* n} x\right\|=\|x\|(n=1,2, \cdots)$. Suppose $y$ is such a vector for $U$ which is orthogonal to the subspaces of $\mathbb{M}$ and $\mathbb{M}^{\perp}$ on which $U$ is unitary. Set $y=y_{M^{\prime}} \oplus y_{M^{\perp}}$ with $y_{M^{\prime}} \in \mathbb{M}$ and $y_{M^{\perp}} \in \mathbb{M}^{\perp}$. By virtue of the Wold decomposition it follows that $\left\|U^{* n} y_{\pi}\right\| \rightarrow 0$ and $\left\|U^{*} y_{n^{\perp}}\right\| \rightarrow 0$. Hence, $y=0$. This concludes the proof.

Various authors [7], [8] have shown that if $S$ is the simple unilateral shift and $U$ is any unitary operator, then there exists a compact operator $K$ such that $S+K$ is unitarily equivalent to $S \oplus U$. On the other hand the present author and J. Pincus [9] have recently shown that if $U$ is any unitary operator with purely singular spectra, then there exists a trace class operator $K$ such that $S+K$ is unitarily equivalent to $S \oplus U$.

\section{REFERENCES}

1. M. Rosenblum, Perturbation of the continuous spectrum and unitary equivalence, Pacific J. Math. 7 (1957), 997-1010. MR 19, 756.

2. T. Kato, Perturbation of continuous spectra by trace class operators, Proc. Japan Acad. 33 (1957), 260-264. MR 19, 1068.

3. S. T. Kuroda, Perturbation of continuous spectra by unbounded operators. I, J. Math. Soc. Japan 11 (1959), 246-262. MR 22 \#8345.

4. T. Kato, Perturbation theory for linear operators, Die Grundlehren der math. Wissenschaften, Band 132, Springer-Verlag, New York, 1966. MR 34 \#3324.

5. M. Schechter, Basic theory of Fredholm operators, Ann. Scuola Norm. Sup. Pisa (3) 21 (1967), 261-280. MR 36 \#6977.

6. B. Sz.-Nagy and C. Foias, Analyse harmonique des opérateurs de l'espace de Hilbert, Masson, Paris; Akad. Kiadó, Budapest, 1967; English rev. transl., North-Holland, Amsterdam; American Elsevier, New York; Akad. Kiadó, Budapest, 1970. MR 37 \#778; 43 \#947. 
7. C. Pearcy and N. Salinas, Compact perturbations of seminormal operators, Indiana Univ. Math. J. 22 (1973), 789-793.

8. L. Brown, R. G. Douglas and P. Fillmore, Unitary equivalence modulo the compact operators and extensions of $C^{*}$-algebras, Lecture Notes in Math., no. 345, Springer-Verlag, Berlin and New York, 1973.

9. R. W. Carey and J. D. Pincus, Unitary equivalence modulo the trace class for self-adjoint operators, Amer. J. Math. (to appear).

DEPARTMENT OF MATHEMATICS, UNIVERSITY OF KENTUCKY, LEXINGTON, KENTUCKY 40506

Current address: Department of Mathematics, State University of New York, Stony Brook, New York 11790 